\title{
Overview of malignant tracheal tumors
}

\author{
Maria Lucia L. Madariaga, Henning A. Gaissert \\ Division of Thoracic Surgery, Department of Surgery, Massachusetts General Hospital, Boston, MA, USA \\ Correspondence to: Henning A. Gaissert. Founders House, Massachusetts General Hospital, 265 Charles Street, Boston, MA 02114-2621, USA. \\ Email: hgaissert@partners.org.
}

\begin{abstract}
Malignant airway tumors present particular challenges for surgeons, namely: to distinguish symptoms from those of the more frequent benign airway diseases; to separate metastatic disease from the uncommon primary tumors; and to consider curative resection in appropriate candidates. Here, we present a critical review of tracheal malignant obstruction, focusing on the evaluation of a patient with malignant airway tumor, patient selection for resection and the predictors of long-term survival. The new development in primary tracheal tumors is an old story, that of making physicians aware that resection rates in epidemiologic studies remain low, mainly because opportunities for resection are missed.
\end{abstract}

Keywords: Trachea; carina; tumor; squamous cell carcinoma (SCC); adenoid cystic carcinoma (ACC)

Submitted Nov 24, 2017. Accepted for publication Feb 27, 2018.

doi: 10.21037 /acs.2018.03.04

View this article at: http://dx.doi.org/10.21037/acs.2018.03.04

\section{Introduction}

Primary tracheal tumors are rare. Among 9,000 autopsies at McGill University and 12,700 autopsies at Montreal General Hospital performed in the 1930s, only two cases of primary tracheal carcinomas were found (1). Based on data from the Netherlands Cancer Registry from 1989 to 2002, the annual incidence of primary tracheal malignancies is 0.142 per 100,000 people; in 15 of 308 cases, the diagnosis was incidentally made at autopsy (2). The largest reported epidemiological study of primary tracheal tumors is based on the Surveillance, Epidemiology, and End Results program (SEER) database of 578 cases from 1973-2004 (3). Among them, $55 \%$ were male and squamous cell carcinoma (SCC) was the predominant histology (45\%) followed by adenoid cystic carcinoma (ACC, 16.3\%), carcinoma not specified or undifferentiated (12.8\%), small cell carcinoma (9.7\%), adenocarcinoma (5.9\%), large cell carcinoma (3.8\%) and sarcoma (3.8\%) (3). One third of cases did not undergo surgery and one quarter underwent procedures ranging from "debulking" to "total removal" or radical surgery, while a majority of cases $(69 \%)$ were treated with radiotherapy (3).

Current epidemiologic and clinical data present conflicting incidence and prevalence for primary malignant airway tumors, for several reasons. While most airway tumors produce symptoms, they are often nonspecific, like shortness of breath, and may not be attributable to the tumors themselves, resulting in diagnostic delay. Further, not every tumor growing in the trachea is of the trachea; lymph nodes bearing metastatic cancer located elsewhere in the lung may progress to the airway lumen and be mistaken for primary disease. Only a careful comparison of radiologic evidence with histologic review separates common metastatic obstruction from rare primary tumors, and few studies provide radiologic or histologic review (2). Finding a large proportion of non-squamous "primary" cancers that are rare in clinical series (for example, adenocarcinoma and small cell carcinoma), in population studies with neither histologic nor radiographic review strongly suggests contamination with metastatic disease. Doubts are therefore appropriate regarding data on resection and survival. Conversely, some tumors prominent in clinical series are far less common in epidemiologic series; the slow-growing ACC, for example, amounts to only $7 \%$ of primary tumors in one epidemiologic study (2), but because of a longer window of time for treatment, occurred as often as SCC in one clinical study (4). A critical appraisal of relevant data confers an attitude of caution toward any unconfirmed diagnosis of primary tracheal tumor. To summarize, 


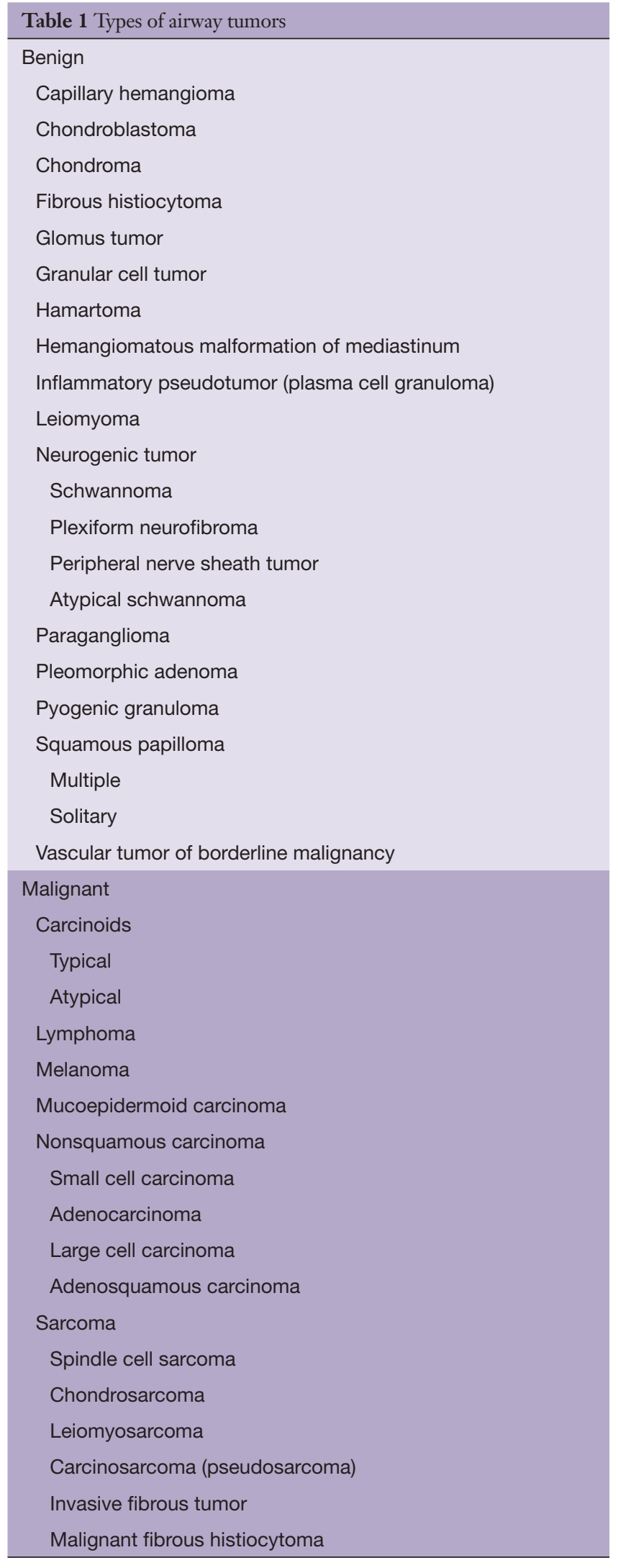

primary tracheal tumors may be even more uncommon than reported in the general population, are rarely considered for surgical resection and remain associated with a poor prognosis when not resected. To surgeons experienced in their treatment, these data suggest underutilization of resection as treatment (5) and a need for better epidemiologic studies.

Primary tracheal tumors in adults are overwhelmingly malignant, whereas in the pediatric population, primary tracheal tumors are usually benign (6). Benign tracheal lesions found in adults include chondroma, granular cell tumor, hamartoma, hemangioma, leiomyoma, neurogenic tumors, pleomorphic adenoma and squamous papillomas (7). The majority of tracheal tumors are secondary endotracheal metastases, occurring via direct invasion from adjacent organs or hematogenous spread (8). This article will focus on malignant primary tracheal tumors in adults (Table 1).

\section{SCC}

The most common primary tracheal malignancy is SCC, comprising about $50-60 \%$ of clinical cases. The histology of these tumors is identical to SCC of the lung. Among 59 cases treated from 1985 to 2008, Honings et al. found that $24 \%$ were well-differentiated, $49 \%$ were moderately differentiated and $27 \%$ were poorly differentiated (9). Unfavorable prognostic factors included tumor extension into the thyroid gland and lymphatic invasion, whereas keratinization, dyskeratosis, necrosis and tumor thickness did not predict prognosis. Progression is marked by extension through the tracheal wall, mediastinal extension or lymph node metastases. Whether lymph nodes in the mediastinum close to the primary tumor portend a similar or worse prognosis than stage N1 disease in lung cancer cannot be derived from current data.

\section{ACC}

ACC is the second-most common primary malignant tracheal tumor, occurring in $10-15 \%$ of cases in epidemiologic studies, but in far greater numbers in clinical series of tracheal resection. They resemble the welldifferentiated, slow-growing tumors of the salivary glands in the neck and may infiltrate in circumferential or longitudinal submucosal planes along the airway axis far beyond the gross tumor, leading to a high rate of positive surgical margins. In a study of 108 consecutive laryngotracheal, tracheal and carinal lesions treated with resection, the tumor was 
intramural in $15 \%$ and extramural in $85 \%$; adjacent organs were invaded in $20 \%$ (10). The cut margins at the airway contained microscopic tumor in $55 \%$, gross tumor in $8 \%$ and were tumor-free in 37\% (10). Late tumor recurrence beyond 10 years after resection may occur.

\section{Other primary malignant tracheal tumors}

As shown in Table 1, less common malignant primary tracheal tumors include carcinoid, lymphoma, melanoma, mucoepidermoid carcinoma and sarcoma (7). Resection is indicated in selected patients with limited disease. Though tracheal invasion by lymphoma is not infrequent, resection is rarely required after chemotherapy and then only for structural instability.

\section{Presentation and diagnosis}

\section{History}

The clinical presentation of tracheal neoplastic obstruction depends on the size of the luminal component, its location and the histologic type and growth pattern. Tracheal tumors large enough to cause symptoms often obstruct half of the airway or decrease the diameter of the tracheal lumen (11). Short-distance obstruction to a tracheal diameter $<8 \mathrm{~mm}$ may result in dyspnea on exertion, whereas an airway of less than $5 \mathrm{~mm}$ typically results in dyspnea at rest (12). In addition to dyspnea, patients may also commonly present with cough, hemoptysis, wheeze, or stridor. In contrast, symptoms related to structures adjacent to the trachea are uncommon. Dysphagia is rare in resectable tumors. Hoarseness may be present when tumors originate close to or within the subglottic space, but hoarseness does not correlate well with vocal cord palsy or recurrent laryngeal nerve invasion (13). Diaphragmatic elevation due to phrenic nerve invasion should be exceedingly rare in resectable tracheal tumors. Patients with SCC usually present in their 7 th decade, whereas patients with ACC are on average 10 years younger (Table 2).

\section{Risk factors}

Patients with SCC commonly have a history of smoking. In non-smokers, history of exposure to alternative carcinogens, such as inhaled hydrocarbons, should be discerned. About $25 \%$ of patients with SCC have a history of prior lung resection for cancer, pointing to the importance of long-
Table 2 Comparison between common malignant primary tracheal tumors SCC vs. ACC

Squamous cell carcinoma (SCC)

Most common primary malignancy of trachea (histology like SCC of lung)

$\mathrm{M}>\mathrm{F}, 60-70$ years

$90 \%$ smokers

Usually exophytic or ulcerative; if membranous tracheal wall involved, consider invasion from primary esophageal tumor

Biologically aggressive, grow rapidly, metastasize early; $30 \%$ have synchronous or metachronous malignancies

Usual diagnosis within 4-6 months of symptom onset

Adenoid cystic carcinoma (ACC)

Arise from bronchial glands (histology like salivary glands)

$\mathrm{M}=\mathrm{F}, 40-50$ years

No smoking association

Usually exerts mass effect rather than regional invasion; submucosal spread

Slow growth, late recurrences

Usual diagnosis more than 1 year after symptom onset

$\mathrm{M}$, male; F, female.

term surveillance bronchoscopy in patients with history of lung cancer at high risk for squamous dysplasia of the large airways (4). There are no known risk factors for ACC.

\section{Delay in diagnosis}

Patients with airway tumors typically have a delay in diagnosis, since symptoms are usually mistaken for other respiratory pathology such as asthma, chronic obstructive pulmonary disease or pneumonia. In our experience, the average duration of delay is 12 months-and this reflects the patient referred to a thoracic surgeon (14). Symptom duration prior to diagnosis is shorter in SCC than ACC. Earlier diagnosis is facilitated by a rapid rate of luminal growth, but in this scenario, the time window for diagnosis in time to apply curative treatment is shorter, as shown by the 3.5 months difference in average symptom duration between resectable and unresectable SCC (14). In addition, $60 \%$ of patients with SCC present with hemoptysis, a symptom difficult to disregard, compared to $30 \%$ of patients with ACC (4). 


\section{Imaging}

Many patients presenting with unannounced malignant airway obstruction had plain chest radiographs early in the course of their illness. The identification of subtle abnormalities in the mediastinal contours or the tracheal air shadow mandates a high index of suspicion for this rare disease; thus, plain films rarely provide the diagnosis, even though abnormalities may become obvious on later review. Computed tomography (CT) with routine axial, coronal and sagittal projections is a precise imaging modality that demonstrates tracheobronchial tumors or extrinsic masses reliably to the systematic reader. In a study of six cases of ACC, CT consistently demonstrated extramural extent of disease; however, the longitudinal extent and invasion of adjacent mediastinal structures was underestimated (15). Recent imaging advances, including multiplanar reformatting and 3D rendering, have improved CT resolution, though the quality of virtual bronchoscopy rendering of CT images does not yet approach clinical bronchoscopy (16). Standard evaluation for locoregional and distant metastatic disease is obtained in bronchogenic carcinoma, ACC and other high-grade malignancies. Positron emission tomography (PET) is helpful in demonstrating the extent of disease for tumors with high radiotracer uptake, such as SCC, while ACC and slowgrowing mucoepidermoid tumors have more variable fluorodeoxyglucose uptake (17).

\section{Spirometry}

There are three characteristic flow volume loop patterns for large airway obstruction: fixed, variable intrathoracic and variable extrathoracic (18). With fixed obstruction, inspiratory and expiratory flow loops have a plateau, reflecting airway resistance that is independent of changes in transmural pressure. Tracheal tumors can also cause a variable intrathoracic obstruction pattern with normal inspiratory loop (negative intrapleural pressure dilates the airway and decreases the degree of obstruction) and an expiratory loop plateau (positive intrapleural pressure narrows the airway and increases the degree of obstruction).

\section{Bronchoscopy}

Every patient found to have malignant airway obstruction, particularly those in distress due to obstruction without diagnosis, should undergo endoscopic evaluation. Rigid bronchoscopy may be used to core out tracheal tumors, providing tissue for histologic diagnosis and aiding in the establishment of a patent airway. Bronchoscopy also provides the most specific information for operative planning, as measurements can be taken to assess whether a tumor is resectable based on its location and size.

\section{Laryngoscopy}

Symptoms suggestive of vocal cord paresis, such as weak voice or aspiration, should also be investigated by a laryngologist to complete the evaluation.

\section{Treatment}

\section{Overview}

The surgeon's contribution to the treatment of malignant airway obstruction due to metastasis is mainly palliative and marginal. Yet their role in the small group of resectable tumors is central to long-term survival. A sensible evaluation demands that patients with limited primary tracheal malignancy are referred to centers and surgeons with experience in the treatment of these tumors. In all regions of the United States, there are now surgeons who are capable of tracheal resection at low risk to the patient, though important limitations persist as these surgeons may not always be known, patients may be reluctant to travel long distances or insurance carriers may create limitations of coverage that prevent referral.

\section{Assessment of resectability}

Tracheal tumors are defined as resectable if the involved tracheal segment can be resected and reconstructed with primary anastomosis. Depending on patient age, weight, neck mobility and comorbidity, approximately half of the trachea may be safely resected. Elderly patients with kyphosis may not tolerate the resection of more than two centimeters of trachea, while individual, young patients with a long, mobile neck may undergo successful reconstruction after resection of six or more centimeters. The quality of this assessment, important for each individual patient, depends on the surgeon's experience. Contraindications to resection of the major airways are respiratory failure, oral corticosteroids and major comorbidity. Diseaserelated contraindications include regional invasion of 
adjacent organs or great vessels, extensive mediastinal lymphadenopathy, distant metastases and previous highdose (>50 Gy) irradiation to the trachea.

Resectability is assessed with imaging and during bronchoscopy, and few patients undergo surgical exploration of the neck or chest alone. The rate of surgical exploration without resection was $6 \%$ at MGH (4). Clinical series suggest that more than half of patients with primary tracheal carcinomas are surgical candidates (19). However, due to the rarity of airway tumors, incorrect diagnosis and suboptimal treatment are common. In a multidisciplinary audit of epidemiologic data from the Netherlands Cancer Registry from 2000 to 2005, 50 cases of primary tracheal carcinoma that were neither metastatic disease nor locally invasive were identified (5). Actual treatment consisted of resection in $12(24 \%)$, radiotherapy in $29(58 \%)$, endobronchial treatment in $6(12 \%)$ and observation in 3 (6\%) (5). The audit identified 16 additional candidates for surgery, suggesting that resection may have been applied in $56 \%$, radiotherapy in $14 \%$ and palliation in $8 \%$ (5). To increase the proportion of surgical resection, patients with tracheal tumors should be referred to a tertiary center with multidisciplinary experience in the treatment of this disease.

\section{Surgical therapy}

\section{Basic principles}

The goal of any surgical intervention should be complete resection. This goal must be balanced with a safe airway anastomosis. The main principles are to protect the lateral blood supply of the trachea, to resect only tracheal or bronchial length that may be safely reconstructed and to avoid excessive anastomotic tension (20). Described here are standard techniques using open incisions. Recent successful adaptation of a thoracoscopic technique in selected patients using spontaneous respiration anesthesia are not considered (21).

\section{Anesthetic management}

Standard intraoperative monitoring includes a peripheral arterial line and bladder catheter. For bronchoscopy, general anesthesia is induced without muscle relaxants, using either inhalational agents or total intravenous anesthesia. Once the airway is secure, muscle relaxation may be administered. Following bronchoscopy, a small-bore endotracheal tube is passed beyond the lesion. During resection and after surgical division of the airway, ventilation is provided across the surgical field by insertion of suitable cuffed tubes or catheters for jet ventilation into the distal airway. Following reconstruction, the orotracheal tube or catheter is reinserted through the anastomosis. A detailed review of anesthetic technique exceeds the purpose of this discussion and is available elsewhere (22). Cardiopulmonary bypass is rarely needed but may be helpful during complex carinal reconstruction.

\section{Positioning for resection}

For tracheal resection via neck or sternum, patients are placed supine with extension of the neck provided by an inflatable cushion underneath the shoulders. The skin is prepped from the chin to the umbilicus. For resection of the lower trachea or carina via right thoracotomy, the patient is placed in typical left lateral decubitus position. Access to the proximal left main bronchus is provided by sternotomy, while the mid and distal left main bronchus is best accessed via left thoracotomy. Bilateral thoracosternotomy is an additional option in selected patients with pericarinal tumors. Resections of the lower larynx, cervical and midtrachea are approached via an anterior cervical collar incision.

\section{Resection technique}

Surgical dissection is performed along the anterior midline plane to mobilize the entire trachea and avoid the recurrent laryngeal nerves, the lateral blood supply of trachea and the esophagus. If the tumor is found to be resectable, circumferential dissection close to the trachea and outside tumor planes is performed. A radical lymph node dissection is avoided since lymph nodes and tracheal blood supply are intimately intertwined; lymph node dissection devascularizes the trachea and precludes anastomotic healing. The trachea is typically divided just distal to the tumor and traction sutures are placed in the lateral wall of the distal trachea. Cross-field ventilation is initiated, intubating the distal trachea with an endotracheal tube or a small catheter for jet ventilation. The segment of trachea containing the tumor is divided cephalad and removed. If doubts about the tolerated length of resection exist, the excision occurs in a segment containing all gross disease, assessing additional excision of microscopic submucosal disease after gauging the extent of tension. If involved, esophageal muscle or thyroid gland 
may be resected en-bloc with the tumor; the surgical soft tissue margins in most tracheal resections are minimal due to the anatomic constraints of the mediastinum.

\section{Intraoperative frozen section analysis}

Separate margins of the divided airway above and below the tumor should be obtained by the surgeon for examination by frozen section unless the limits of safe reconstruction have been reached and additional resection is ruled out. As explained above, incomplete resection is common in ACC given its insidious submucosal spread. Adjuvant postoperative radiation is used to address these margins. Spare sampling of lymph nodes completes the local resection.

\section{Reconstruction technique}

Following circumferential placement of interrupted sutures using absorbable vicryl or polydioxanone, the anastomosis is completed. To reapproximate the airway, the neck is flexed in cooperation with the anesthesiologist to alleviate excessive anastomotic tension. Release maneuvers are used when tension at the anastomosis is excessive. Only about 6\% of patients undergoing primary resection require release maneuvers (23). The most common maneuver to gain additional tracheal length is the Montgomery suprahyoid release (24). Suprahyoid release is helpful for gaining upper tracheal mobility, as severing muscular attachments and lateral segments of the central hyoid results in downward displacement of larynx and cervical trachea by $1-2 \mathrm{~cm}$. However, this release maneuver often results in temporary postoperative dysphagia. Intrathoracic tracheal mobilization by circumcision of the pericardium around the pulmonary veins may be used liberally to gain additional length for reconstruction of the lower trachea (25).

\section{Carinal resection}

Carinal resections offer a unique set of problems and carry the highest risk of any tracheobronchial resection. In 1982, Grillo et al. published a series of 36 carinal resections detailing options for primary resection with carinal reconstruction, with or without various amounts of pulmonary resection (26). Twenty-three resections in this series were performed for primary neoplasm of the airway (14 ACC, 4 carcinoids, 2 SCC and 1 each of mucoepidermoid carcinoma, myxoid spindle cell sarcoma, granular cell tumor). The perioperative mortality was 13\% (1 from erosion into the pulmonary artery and 3 from respiratory failure) and $8 \%$ had stenosis at the anastomosis (26). Mitchell and associates' follow-up paper in 1999 encompassed the largest clinical experience with carinal resection with 143 cases (27). The importance of reducing anastomotic tension was pointed out, as 64 patients (45\%) underwent tracheal release maneuvers, including 49 hilar pericardial releases, 3 laryngeal releases and a combination in 12 cases (27). Laryngeal release was of no use in carinal reconstruction. There were 15 different modes of reconstruction based on the type and extent of resection: 52 patients had undergone carinal resection without pulmonary resection; 68 patients had undergone carinal resection plus pneumonectomy; and 14 patients had undergone carinal resection plus lobar resection (27). Because the left mainstem bronchus is fixed by the aortic arch, downward mobilization of the trachea is more important on this side. Occasionally, after maximal release maneuvers, anastomosis of the right mainstem bronchus to the trachea and the left mainstem bronchus to the right mainstem bronchus or the bronchus intermedius was necessary to reduce tension.

\section{Postoperative care}

Postoperative care consists of initial observation in a nursing unit experienced in airway recovery. Oral intake is limited during the first 3 to 4 days, and swallowing is evaluated and supervised thereafter. Neck extension is limited with the use of a suture between the chin and anterior chest that must be loose enough to prevent neurologic injury due to forced neck flexion. The anastomosis is checked with the bronchoscope after one week. If delayed healing is observed, discharge from the hospital is prolonged. In a recent report, five patients with failed anastomotic healing (from necrosis to partial separation) were treated daily or twice daily with hyperbaric oxygen for 90 minutes via a hyperbaric oxygen chamber pressured to $2 \mathrm{~atm}$ with $100 \%$ oxygen (28). After 5 to 14 days of treatment, all patients had evidence of anastomotic healing on bronchoscopy and none required tracheostomy, T-tube or re-operation (28). After discharge, all patients are advised to limit neck extension or rotation for 1 month.

\section{Surveillance}

While ACC is associated with late local recurrence and metastases, second primary SCC may continue to arise 
elsewhere in the airway. We and others suggest endoscopic surveillance, though options for second interventions are limited given the use of adjuvant high-dose radiation.

\section{Adjuvant therapy}

\section{Radiation}

Adjuvant radiation is administered with the recognition that local disease control provided by surgical resection is imperfect. A majority of primary malignant tracheal tumors, including the most common carcinomas of SCC and ACC, extend to extramural tissues where surgical margins are slim. For this reason, and in the absence of any prospective evidence, there is widespread, though sparsely published, agreement to add postoperative mediastinal radiation following successful tracheal and carinal resections for locally advanced tumors, excluding only the earliest lesions. Success is defined here as a completely healed anastomosis. Because the effect of anastomotic tension persists for some time, patients are offered adjuvant therapy at least 2 months after their operation, or later if there are concerns about the integrity of the anastomosis. The tumor types selected for radiation include all bronchogenic carcinomas, ACC and soft tissue sarcoma. On the basis of prior experience, a dose of 54 Gy is administered. When intensity-modulated radiotherapy (IMRT) is administered, the dose may reach $60 \mathrm{~Gy}$. In randomized trials of patients who underwent resection of advanced head and neck cancers, patients who received postoperative chemoradiation had improved survival, decreased local recurrence, but higher adverse effects than patients who received adjuvant radiation alone $(29,30)$. Particularly in cases where surgical resection was limited due the length of tracheal disease, postoperative radiotherapy should be considered. Of note, $59 \%$ of patients with ACC in the MGH experience had positive tracheal margins in contrast to $18 \%$ of patients with SCC (31). Apart from positive margins, other high-risk features that should prompt adjuvant therapy include advanced tumor stage (T3, T4), extracapsular extension, perineural or lymphovascular invasion. Patients with low-grade tumors, such as carcinoid or localized, well-differentiated mucoepidermoid carcinoma, do not require adjuvant radiation (31). A retrospective matched-pair analysis of the SEER database was performed to determine whether radiation therapy improves outcomes in patients with malignant primary tracheal tumors (32). Patients who received radiation between 1988 and 2007 were matched to patients with similar demographics, tumor histology, extent of disease and surgical resection who did not receive radiation therapy. Treatment with radiation was associated with improved survival, particularly in patients with SCC, regional disease extension, or in patients with unresectable disease (32). In the Netherlands Cancer Registry, patients who underwent resection without radiotherapy had a median survival of 91 months, patients who underwent resection with radiotherapy had a median survival of 82 months, patients who underwent radiotherapy alone had a median overall survival of 11 months and in patients who did not receive either therapy, median survival time was 3 months (2).

\section{Chemotherapy}

Postoperative adjuvant cisplatin-based chemotherapy may be considered in bronchogenic carcinoma with concurrent radiation therapy, in reference to the treatment of more peripheral lung cancer. More study is needed to see whether chemotherapy provides benefit.

\section{Unresectable tumors}

\section{Overview}

Endoscopic options for managing malignant airway obstruction, such as use of lasers and stents, are palliative and reserved for unresectable tumors or patients with contraindications to surgery. In a series of 2,008 patients from Italy with malignant airway obstruction, 1,838 patients underwent 2,610 laser resections, 306 patients underwent placement of 393 tracheobronchial silicone stents and 66 patients underwent endoluminal brachytherapy (33). Quality of life improved in $93 \%$ of patients undergoing laser resection, but palliation is usually temporary (33).

\section{Stents and T-tubes}

Stents, specifically self-expanding metal mesh stents, should not be considered a "bridge" to surgery, as their use is associated with local complications. Stents should only be employed for patients who are not candidates for resection. Self-expanding metal stents may be used in patients with expected 3-6-month survival; drawbacks to these stents include dislodgement, formation of esophageal and vascular fistulas and stricture formation above and below the stent (31). Left in the trachea long enough, virtually every self-expanding tracheal stent will result in a complication; thus, they are 


\begin{tabular}{|c|c|c|c|c|}
\hline Grillo et al. (36) & $1962-1989$ & $198(\mathrm{MGH})$ & SCC, $36 \% ;$ ACC, $40 \%$ & SCC, $45 \%^{*}$; ACC, $65 \%$ * \\
\hline Gaissert et al. (4) & $1962-2002$ & 270 (MGH) & SCC, $50 \%$; ACC, $50 \%$ & $\begin{array}{l}\text { SCC resectable, 39\%; SCC unresectable, } 7 \% \text {; } \\
\text { ACC resectable, 52\%; ACC unresectable, 33\% }\end{array}$ \\
\hline Honings et al. (2) & 1989-2002 & $\begin{array}{l}308 \text { (Netherlands } \\
\text { Cancer Registry) }\end{array}$ & SCC, $53 \%$; ACC, $7 \%$ & SCC, $12 \% ;$ ACC, $61 \%$ \\
\hline Urdaneta et al. (3) & 1973-2004 & 573 (SEER database) & SCC, $45 \%$; ACC, $16 \%$ & SCC, $13 \% ;$ ACC, $74 \%$ \\
\hline
\end{tabular}

unsuitable for unresectable ACC-patients with this tumor may survive for years. Longer-term palliation may be more effectively managed with a silicone T-tube that results in limited damage to normal tracheal wall.

\section{Chemoradiation}

Palliative radiation may also be offered for management of airway obstruction symptoms. Because of the rarity of airway tumors, there are no clinical trials that define the optimum approach for unresectable or metastatic SCC or ACC. Current practice includes platinum-based chemoradiation in selected patients with bronchogenic carcinoma $(34,35)$.

\section{Outcomes}

\section{Overview}

Prognostic factors predicting longer survival are tumor histology (ACC better than SCC and other bronchogenic carcinoma) and resection status (complete resection with negative margins) (Table 3). Operative mortality and complications have decreased over time.

\section{Tumor histology and resectability}

Negative prognostic factors for overall survival after resection of airway tumors include incomplete resection, invasion into adjacent structures (thyroid gland) or extramural disease, lymphatic invasion or lymphatic metastases, and perineural growth $(9,10)$. The French Society of Cardiovascular Surgery published a retrospective review of 208 patients who underwent resection for primary tracheal tumors from 1970 to 1993 (37). Among the 26 included surgical centers, only ten teams operated on ten patients or more. Survival was better in patients with ACC and in patients who underwent complete resection (37). In a review of 38 patients with ACC from 1963 to 1995, the Toronto group found that mean survival after resection was 7.5 years and $50 \%$ of resections had residual tumor at the airway margin on final pathologic examination (38). Our institution found similar results. Mean survival after resection of tumors with positive microscopic margins was 2.1 years in patients with SCC, versus 13.3 years in patients with ACC (6.1 years in patients with ACC with grossly positive margins) $(9,10)$. Mean survival after resection of tumors with lymphatic invasion was 4.6 years in patients with SCC versus 6.1 years in patients with ACC $(9,10)$. A retrospective study of 270 patients at our institution from 1962 to 2002 showed that in patients with ACC who underwent resection, overall 5 - and 10-year survival was $52 \%$ and $29 \%$, respectively, in contrast to $33 \%$ and $10 \%$ for unresectable disease (4). In patients with SCC who underwent resection, overall 5 - and 10-year survival was $39 \%$ and $18 \%$, while only $7 \%$ and $5 \%$ survived with unresectable disease (4). Though SCC was associated with higher postoperative mortality and poorer long-term results, ACC was associated with late local recurrence and the appearance of metastases even after 10 years.

In a retrospective review of 308 cases of primary tracheal tumors from the Netherlands Cancer Registry (1989-2002), median survival was 10 months and 1-, 5-, and 10-year survival rates were $43 \%, 15 \%$ and $6 \%$, respectively (2). Among surgical patients, 5- and 10-year survival was 51\% and $33 \%$, respectively (2). In contrast, 5 -year survival among patients receiving radiation therapy alone was $11 \%$ and among patients who received no therapy, $3 \%$ (2). In the SEER database of 578 cases of primary tracheal 


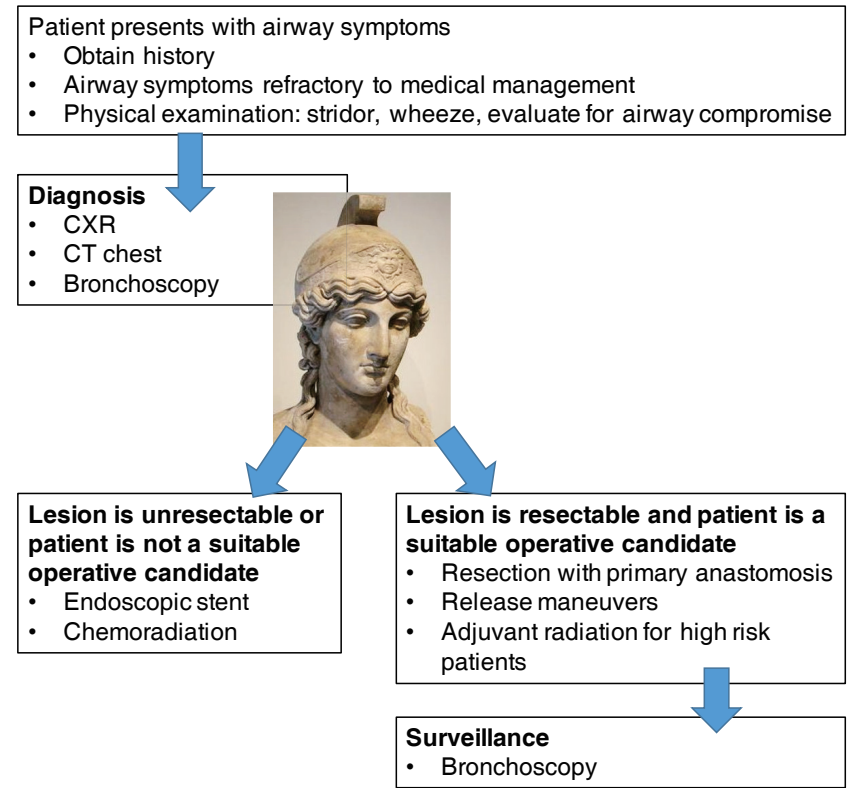

Figure 1 Approach to evaluation of malignant primary tracheal tumor with the head of Athena representing clinical judgement. CXR, chest X-ray; CT, computed tomography.

tumors [1973-2004], overall 5-year survival was 27\%; patients with ACC had better 5-year survival (74.3\%) than SCC (12.6\%) (3). Other factors associated with improved survival were surgical intervention and localized disease. Treatment with radiation, with or without surgery, did not affect survival (3). These data suggest that results after surgery are better than with radiation alone; however, these retrospective studies are limited because unresectable patients are included in the radiation alone group, which skews this group towards a worse outcome.

\section{Postoperative mortality and complications}

In a single institution retrospective study of 270 patients with ACC or SCC from 1962 to 2002, overall operative mortality improved over time, from $21 \%$ during the first decade to $3 \%$ during the fourth decade (4). Postoperative complications after tracheal reconstruction are not common but can cause significant morbidity. Complications at the anastomosis include granulation, restenosis and dehiscence. Anastomotic granulation has largely disappeared with the present-day use of absorbable sutures for the anastomosis. In the largest series of patients who underwent tracheal resection at our institution (of whom $23 \%$ underwent resection for tracheal tumors), $95 \%$ of 901 patients had a "good or excellent" airway result while $4 \%$ of patients remained with permanent tracheostomy or T-tube. The mortality was $1 \%$ (39). The Grillo classification of airway outcome defines "excellent" as normal airway anatomy without symptoms, "good" as narrowed anatomy without symptoms, "satisfactory" as sufficient to perform daily activities but limitations during major physical activities and "failure" as all other outcomes. Anastomotic complications occurred in $9 \%$ of patients and were treated with multiple dilations (2.4\%), temporary tracheostomy (8.6\%), temporary T-tube $(19.8 \%)$, permanent tracheostomy $(17.2 \%)$, permanent T-tube (25\%) or reoperation (20\%) (39). Factors predicting anastomotic complications were reoperation [odds ratio (OR), 3.0], diabetes (OR, 3.3), length of resection $\geq 4 \mathrm{~cm}$ (OR, 2.01), laryngotracheal resection (OR, 1.8), age 17 years or younger $(\mathrm{OR}, 2.3)$ and need for preoperative tracheostomy (OR, 1.8) (39). In the Mitchell series of carinal resection, postoperative mortality was $12.7 \%$ while $39 \%$ of patients had postoperative complications, including the anastomosis $(17 \%)$, arrhythmias $(15 \%)$ and pneumonia (8\%) (27).

Anastomotic dehiscence represents the worst outcome after tracheal and carinal reconstruction, with dire consequences particularly in the setting of the latter. Dehiscence often leads to local mediastinitis and progresses to pneumonia and respiratory insufficiency. After tracheal resection, dehiscence may cause a tracheoinnominate fistula. Securing the airway is most challenging after failed carinal reconstruction, since lower airway anastomoses are more difficult to stent. Often, at least a temporary tracheostomy or T-tube is needed.

\section{Conclusions}

In this critical review of malignant tracheal obstruction, we emphasize the role of clinical judgement in diagnosis and selection of patients for curative resection (Figure 1). Strategies to decrease tumor burden with targeted therapy or to create alternative conduits by tracheal transplantation or bioengineering may, in the future, increase therapeutic options available to patients with malignant airway tumors and improve survival outcomes.

\section{Acknowledgements}

None. 


\section{Footnote}

Conflicts of Interest: The authors have no conflicts of interest to declare.

\section{References}

1. Ellman P, Whittaker H. Primary carcinoma of the trachea. Thorax 1947;2:153-62.

2. Honings J, van Dijck JA, Verhagen AF, van der Heijden $\mathrm{HF}$, Marres HA. Incidence and treatment of tracheal cancer: a nationwide study in the Netherlands. Ann Surg Oncol 2007;14:968-76.

3. Urdaneta AI, Yu JB, Wilson LD. Population based cancer registry analysis of primary tracheal carcinoma. Am J Clin Oncol 2011;34:32-7.

4. Gaissert HA, Grillo HC, Shadmehr MB, et al. Longterm survival after resection of primary adenoid cystic and squamous cell carcinoma of the trachea and carina. Ann Thorac Surg 2004;78:1889-96; discussion 1896-7.

5. Honings J, Gaissert HA, Verhagen AF, et al. Undertreatment of tracheal carcinoma: multidisciplinary audit of epidemiologic data. Ann Surg Oncol 2009;16:246-53.

6. Junker K. Pathology of tracheal tumors. Thorac Surg Clin 2014;24:7-11.

7. Gaissert HA, Grillo HC, Shadmehr MB, et al. Uncommon primary tracheal tumors. Ann Thorac Surg 2006;82:26872; discussion 272-3.

8. Madariaga ML, Gaissert HA. Secondary tracheal tumors: a systematic review. Ann Cardiothorac Surg 2018;7:183-96.

9. Honings J, Gaissert HA, Ruangchira-Urai R, et al. Pathologic characteristics of resected squamous cell carcinoma of the trachea: prognostic factors based on an analysis of 59 cases. Virchows Arch 2009;455:423-9.

10. Honings J, Gaissert HA, Weinberg AC, et al. Prognostic value of pathologic characteristics and resection margins in tracheal adenoid cystic carcinoma. Eur J Cardiothorac Surg 2010;37:1438-44.

11. Brand-Saberi BE, Schafer T. Trachea: anatomy and physiology. Thorac Surg Clin 2014;24:1-5.

12. Sherani K, Vakil A, Dodhia C, et al. Malignant tracheal tumors: a review of current diagnostic and management strategies. Curr Opin Pulm Med 2015;21:322-6.

13. Gaissert HA, Grillo HC, Shadmehr BM, et al. Laryngotracheoplastic resection for primary tumors of the proximal airway. J Thorac Cardiovasc Surg 2005;129:1006-9.
14. Gaissert HA, Burns J. The compromised airway: tumors, strictures, and tracheomalacia. Surg Clin North Am 2010;90:1065-89.

15. Spizarny DL, Shepard JA, McLoud TC, et al. CT of adenoid cystic carcinoma of the trachea. AJR Am J Roentgenol 1986;146:1129-32.

16. Kligerman S, Sharma A. Radiologic evaluation of the trachea. Semin Thorac Cardiovasc Surg 2009;21:246-54.

17. Park CM, Goo JM, Lee HJ, et al. Tumors in the tracheobronchial tree: CT and FDG PET features. Radiographics 2009;29:55-71.

18. Hyatt RE. Evaluation of major airway lesions using the flow-volume loop. Ann Otol Rhinol Laryngol 1975;84:635-42.

19. Gaissert HA. Primary tracheal tumors. Chest Surg Clin N Am 2003;13:247-56.

20. Madariaga ML, Gaissert HA. Reresection for recurrent stenosis after primary tracheal repair. J Thorac Dis 2016;8:S153-9.

21. Li J, Liu H, Liu J, et al. Challenges in complex videoassisted thoracoscopic surgery and spontaneous respiration video-assisted thoracoscopic surgery procedures. J Vis Surg 2017;3:31.

22. Hobai IA, Chhangani SV, Alfille PH. Anesthesia for tracheal resection and reconstruction. Anesthesiol Clin 2012;30:709-30.

23. Donahue DM, Grillo HC, Wain JC, et al. Reoperative tracheal resection and reconstruction for unsuccessful repair of postintubation stenosis. J Thorac Cardiovasc Surg 1997;114:934-8; discussion 938-9.

24. Montgomery WW. Suprahyoid release for tracheal anastomosis. Arch Otolaryngol 1974;99:255-60.

25. Grillo HC, Dignan EF, Miura T. Extensive Resection and Reconstruction of Mediastinal Trachea without Prosthesis or Graft: An Anatomical Study in Man. J Thorac Cardiovasc Surg 1964;48:741-9.

26. Grillo HC. Carcinoma of the lung: what can be done if the carina is involved? Am J Surg 1982;143:694-5.

27. Mitchell JD, Mathisen DJ, Wright CD, et al. Clinical experience with carinal resection. J Thorac Cardiovasc Surg 1999;117:39-52; discussion 52-3.

28. Stock C, Gukasyan N, Muniappan A, et al. Hyperbaric oxygen therapy for the treatment of anastomotic complications after tracheal resection and reconstruction. J Thorac Cardiovasc Surg 2014;147:1030-5.

29. Bernier J, Domenge C, Ozsahin M, et al. Postoperative irradiation with or without concomitant chemotherapy for locally advanced head and neck cancer. N Engl J Med 
2004;350:1945-52.

30. Cooper JS, Pajak TF, Forastiere AA, et al. Postoperative concurrent radiotherapy and chemotherapy for high-risk squamous-cell carcinoma of the head and neck. N Engl J Med 2004;350:1937-44.

31. Gaissert HA, Honings J, Gokhale M. Treatment of tracheal tumors. Semin Thorac Cardiovasc Surg 2009;21:290-5.

32. Xie L, Fan M, Sheets NC, et al. The use of radiation therapy appears to improve outcome in patients with malignant primary tracheal tumors: a SEER-based analysis. Int J Radiat Oncol Biol Phys 2012;84:464-70.

33. Cavaliere S, Venuta F, Foccoli P, et al. Endoscopic treatment of malignant airway obstructions in 2,008 patients. Chest 1996;110:1536-42.

34. Joshi NP, Haresh KP, Das P, et al. Unresectable basaloid squamous cell carcinoma of the trachea treated with concurrent chemoradiotherapy: a case report with review of literature. J Cancer Res Ther 2010;6:321-3.

Cite this article as: Madariaga ML, Gaissert HA. Overview of malignant tracheal tumors. Ann Cardiothorac Surg 2018;7(2):244-254. doi: 10.21037/acs.2018.03.04
35. Allen AM, Rabin MS, Reilly JJ, et al. Unresectable adenoid cystic carcinoma of the trachea treated with chemoradiation. J Clin Oncol 2007;25:5521-3.

36. Grillo HC, Mathisen DJ. Primary tracheal tumors: treatment and results. Ann Thorac Surg 1990;49:69-77.

37. Regnard JF, Fourquier P, Levasseur P. Results and prognostic factors in resections of primary tracheal tumors: a multicenter retrospective study. The French Society of Cardiovascular Surgery. J Thorac Cardiovasc Surg 1996;111:808-13; discussion 813-4.

38. Maziak DE, Todd TR, Keshavjee SH, et al. Adenoid cystic carcinoma of the airway: thirty-two-year experience. J Thorac Cardiovasc Surg 1996;112:1522-31; discussion 1531-2.

39. Wright CD, Grillo HC, Wain JC, et al. Anastomotic complications after tracheal resection: prognostic factors and management. J Thorac Cardiovasc Surg 2004;128:731-9. 